\title{
complicità
}

\section{Complicità e responsabilità reciproca}

Carla Bagnoli

ABSTRACT: This essay criticizes theories which conceive of complicity in terms of causal, intentional contribution, or treat non-ideal circumstances as mitigating conditions. By contrast, the essay argues that complicity is a personal modality rooted in interdependence, which is a constitutive feature of human rational agency. Interdependence implicates us in a broad range of collective phenomena, including large scale harms. This diagnosis points out that we can rely on normative and practical resources for building new forms of shared agency. To this effect, moral principles should be reconceived as functional to practical rationality, rather than adapted and compromised to fit non-ideal conditions.

KEYWORDS: Complicity, interdependence, collective agency, practical rationality

\section{Introduzione}

La complicità sembra richiedere un qualche contributo all'azione di un agente primario, ma è una questione aperta di che natura sia questo contributo e se il complice sia esso stesso un agente. Il mio intento in questo articolo è esplicativo e normativo. In primo luogo, cerco di far emergere che la complessità e varietà dei fenomeni associati alla complicità rivela che si tratta di una categoria composita, nella quale il contributo del complice può essere identificato almeno in tre modi: (i) come contributo causale alla produzione di certi effetti, (ii) come contributo alla partecipazione intenzionale all'attività dell'agente, oppure (iii) come una relazione normativa specifica che lega il complice all'agente primario attraverso il concorso nell'azione, che si può estendere alle forme di interdipendenza. Dal punto di vista normativo, propongo di concepire la complicità all'interno delle relazioni di interdipendenza, per sostenere l'ineludibilità delle responsabilità morali nelle condizioni di ingiustizia sistemica nelle quali ci troviamo. L'argomento è articolato nel modo seguente.

Prima di entrare nel vivo della discussione, offro una raccomandazione di metodo volta a favorire una caratterizzazione della complicità articolata su un ampio spettro di esempi paradigmatici. Poi presento alcuni esempi

Articolo ricevuto il $1^{\circ}$ ottobre 2020, accettato il 29 ottobre 2020. Affiliazione: Università degli Studi di Modena e Reggio Emilia. Email: carla.bagnoli@unimore.it 
ordinari nei quali la complicità si manifesta come un atteggiamento personale piuttosto che come un intervento di tipo causale e intenzionale. Rilevo alcune difficoltà del modello standard che identifica la complicità attraverso il contributo causale del complice. Passo poi all'esame la teoria secondo la quale la complicità comporta un contributo intenzionale, ovvero, l'intenzione a partecipare all'azione. Mi soffermo, in particolare, su alcuni esempi di complicità che mostrano il carattere parziale del modello intenzionalista. Infine, esamino criticamente la proposta teorica che si incentra sull'identità pratica di ruolo. Essa si incardina su un argomento che sfrutta la distinzione tra condizioni ideali e non-ideali, per sostenere che le condizioni non-ideali fungono da circostanze attenuanti e costringono a mitigare il rigore dei principi morali. In contrasto con questo argomento, sostengo che la complicità è una modalità relazionale strutturale caratteristica di agenti razionali mutualmente dipendenti. L'interdipendenza crea opportunità di azione e ci implica reciprocamente nel concorso di fenomeni di larga scala. Ciò significa che non ci possiamo sottrarre all'accusa di complicità, ma anche che abbiamo risorse normative non ancora pienamente utilizzate che, come la complicità, sono radicate nella costitutiva interdipendenza. In questa prospettiva, i principi morali devono essere ripensati nella loro funzionalità di principi di razionalità pratica, anziché adattati alla moralità compromissoria delle circostanze non-ideali.

\section{Complicità a spettro largo}

In molti codici di diritto penale, la complicità non è un tipo di crimine, ma un modo di commettere un crimine, è una dottrina che specifica quando si è legalmente punibili per un crimine compiuto da un'altra persona. Le implicazioni sono molto complesse, anche perché questa definizione si presta a strumentalizzazioni e può facilmente essere usata come mezzo per evitare la responsabilità penale, incolpando un altro agente per i crimini commessi ${ }^{1}$.

Se nella giurisprudenza la nozione di complicità ha una formulazione sufficientemente chiara da consentire una tassonomia di base utilizzabile da un sistema normativo coerente, la trattazione filosofica di questa nozione appare frammentaria e dispersiva, sia per gli aspetti che vengono messi in luce, sia per le direzioni teoretiche e normative che le analisi filosofiche della complicità hanno ispirato. Ma forse queste caratteristiche di frammentarietà e multi-direzionalità ci danno una indicazione metodologica importante: se

1 Vd. C. Lepora and R. E. Goodin, On complicity and compromise, Oxford University Press, Oxford 2013. 
abbandoniamo il proposito positivo-normativo (ovvero la necessità del legislatore di definire in modo perspicuo il fenomeno che ha da essere normato, e quindi il compito del giurista che esamina la coerenza tra la lettera della legge e la volontà del legislatore, e ne considera l'applicabilità), il compito della filosofia potrebbe essere ricostruttivo e propositivo. L'esplorazione filosofica ha compiti meta-normativi e quando questi riguardano i criteri di correttezza delle norme, si tratta di criteri normativi di altro livello rispetto a quelli studiati e applicati dal giurista.

L'esplorazione filosofica, a mio avviso, deve iniziare dalla considerazione della pluralità di esempi paradigmatici che aiutano a chiarire quali fenomeni appartengono alla categoria filosofica di complicità. Questa categoria, così costruita, non coincide necessariamente con la categoria che le tradizioni del diritto ci mettono a disposizione e proprio per questo può rappresentare la base per un confronto critico. Il mio primo compito è di far emergere che la complessità e varietà dei fenomeni associati alla complicità rivela che si tratta di una categoria composita, nella quale il contributo del complice può essere identificato almeno in tre modi: (i) come contributo causale alla produzione di certi effetti, (ii) come contributo alla partecipazione intenzionale all'attività dell'agente, oppure (iii) come una relazione normativa specifica che lega il complice all'agente primario attraverso il concorso nell'azione. Quest'ultima caratterizzazione mi sembra adeguata a un'ampia sezione di fenomeni che non hanno attratto l'attenzione dei filosofi. Questa disattenzione è, almeno in parte, motivata dal fatto che la complicità è stata discussa principalmente in relazione a crimini e azioni immorali individuali, e ritagliata sulla categoria del diritto penale, con particolare riferimento ai crimini collettivi e ai contesti di ingiustizia normalizzata. Tuttavia, allargando il quadro di osservazione a casi ordinari di complicità nell'azione emergono aspetti della relazione di complicità che ci aiutano a comprendere meglio anche fenomeni di complicità già categorizzati.

\section{La complicità come atteggiamento personale}

Si può iniziare dal fatto che in un senso del tutto ordinario la complicità non riguarda l'agire nel suo aspetto esecutivo e performativo, ma ha i connotati dell'atteggiamento personale, ovvero che una persona intrattiene con un'altra persona, piuttosto che una modalità di operare. Si tratta di un atteggiamento che determina un certo tipo di deliberazione pratica e che ha a che fare con l'agire, ma appunto nel contesto di interazioni personali. Per esempio, guardando all'atteggiamento coordinato di due gemelli che giocano nel parco, si può osservare il loro sguardo complice, un particolare atteggiamento di intimità e fiducia reciproca, che li unisce e al contempo li 
separa dal contesto. Questo sguardo guida immediatamente i loro gesti: senza ricorrere ad accordi verbali su chi deve fare che cosa, i due si spartiscono senza discutere giochi e premi. Si tratta di azioni deliberate, dove la complicità ha preso il posto della deliberazione discorsiva, procedurale. Oppure, ecco una anziana coppia di fronte al cartellone di un teatro, uno sguardo d'intesa ed entrano a comprare i biglietti per il concerto di András Schiff. Come nell'esempio precedente, la decisione di acquistare i biglietti è deliberata, eppure non è il risultato di alcuna negoziazione a proposito di alcunché: è stata sufficiente la complicità. Si può immaginare che questa sia una relazione costruita nel tempo, forse faticosamente, attraverso aggiustamenti e compromessi. Quale che sia la sua storia, questa relazione si dimostra normativa, ovvero, capace di dirigere l'attenzione, generando un universo condiviso di salienze e quindi proponendo ragioni per l'azione che si impongono con un'autorità immediata - ma immediata in quanto condivisa.

Questi due esempi presentano una componente psicologica, normativa e relazionale della complicità che fa parte della rappresentazione ordinaria delle nostre interazioni con l'altro. Lo sguardo complice è una forma di attenzione congiunta che rappresenta uno dei meccanismi psicologici più importanti non solo nelle forme di socializzazione diadica esemplificate sopra, ma anche nella formazione di abilità sociali e interpretative che sono alla base delle risposte emotive e sociali degli agenti individuali e che si manifestano in una comprensione del mondo esterno come ambiente condiviso ${ }^{2}$. È un fenomeno comune e riconoscibile eppure non è chiaro in quale relazione esso sia con la complicità come categoria della teoria dell'azione e della filosofia morale e politica, che è intesa in un senso strettamente individualistico. Inoltre, nelle trattazioni filosofiche, la complicità ricopre un ruolo centrale solo nella misura in cui si rintraccia un contributo causale o intenzionale del complice, ciò che emerge mettendo a fuoco l'aspetto esecutivo e performativo dell'agire. Ritengo che questo dipenda dal fatto che la categoria filosofica della complicità è ritagliata su quella giuridica ed è motivata dalla preoccupazione di precisare la base di una imputazione individuale rispetto a un atto performativo.

2 Vd. C. Bagnoli \& C. Calabi, Attenzione congiunta e salienze condivise, "Nuova Civiltà delle Macchine", 23, 2005/1, pp. 35-48; J. Campbell, Joint attention, in M. Jankovic \& K. Ludwig (Eds.), The Routledge handbook of collective intentionality, Routledge, New York 2018, pp. 115-129; Cfr. anche D. Zahavi, You, Me, and We: The Sharing of Emotional Experiences, "Journal of Consciousness Studies", 22, 2015, pp. 84-101. 


\section{L'insufficienza del paradigma causale}

Questa postura teorica è apparente nelle teorie causaliste che identificano la complicità nei termini di un contributo causale, seppure secondario rispetto al ruolo dell'agente primario dell'azione ${ }^{3}$. Ma la tendenza a interpretare la complicità nel senso di un contributo causale è incoraggiata anche da certe teorie etiche, ovvero, quelle che adottano un criterio consequenzialista di rilevanza morale ${ }^{4}$. Il consequenzialismo presenta una difficoltà a mio avviso fatale. Da una parte, se l'aspetto morale dell'agire è identificato in termini delle conseguenze che vengono prodotte attraverso il contributo causale dell'agente, se ne ricava una concezione piuttosto angusta di complicità, incapace di rendere conto di quei casi paradigmatici nei quali l'agente è complice in un senso psicologico che non si traduce facilmente in termini di un contributo causale all'azione. Per esempio, in caso di istigazione, un agente può essere considerato complice in quanto induce psicologicamente al crimine, ma non svolge un ruolo causale nel senso che non 'determina' l'altro a compiere un atto preciso. Si potrebbe ribattere che la manipolazione o l'influenza psicologica è di tipo causale, ma ciò che rimane da spiegare è l'autorità che un agente esercita sull'altro nei processi di asservimento e strumentalizzazione, ovvero, quando lo rende complice o servo della sua azione. Il paradigma causale standard è fuorviante perché interpreta la relazione personale che genera la complicità interamente nei termini di causa ed effetto. Ma il contributo causale è solo uno dei modi in cui si può concorrere a generare l'azione. Inoltre, vi sono modalità della complicità in cui il contributo non è facilmente comprensibile nemmeno come concorso in azione.

Si può obiettare che se un agente non contribuisce in qualche modo alla produzione di un'azione, è difficile dire in che senso ne è complice ${ }^{5}$. D'altra parte ci sono casi in cui l'agente si rende complice di un misfatto dopo che

\footnotetext{
3 Per esempio, John Gardener intende la complicità come partecipazione nell'azione malvagia di un altro agente, vd. Complicity and Causality, “Criminal Law and Philosophy”, 1, 2007, pp. 127-141. L'unico modo di partecipare è l'interazione causale, quindi il complice contribuisce attraverso il contributo causale. Tuttavia, c'è una distinzione importante tra agente primario e complice, che è fondamentale per la comprensione della struttura dell'agire.

4 Per una giustificazione consequenzialista della complicità, vd. S. Kagan, Do I make a difference?, "Philosophy \& Public Affairs", 39, 2011, pp. 105-141. Contro il paradigma consequenzialista, invece, vd. C. Kutz, Causeless complicity, "Criminal Law and Philosophy", 1, 2007/3, pp. 289-305, e M. B. Budolfson, The inefficacy objection to consequentialism and the problem with the expected consequences response, "Philosophical Studies", 176, 2019, pp. 1711-1724; T. Isaacs, Moral Responsibility in Collective Contexts, Oxford University Press, Oxford 2011; J. Nefsky, How you can help, without making a difference, "Philosophical Studies", 174, 2017, pp. 2743-2767.

5 Questa intuizione è esplicitata da G. Mellema, Complicity and Moral Accountability, University of Notre Dame Press, Indiana 2016, p. 68.
} 
ciò è avvenuto, per esempio non denunciandolo oppure coprendo l'agente primario. Prendiamo il caso di un collega che ripetutamente scredita l'operato degli altri membri del team per far risaltare il suo e si attribuisce meriti che non ha avuto. Il direttore di ricerca viene a sapere di questi episodi, ma non vuole assumersi la responsabilità di intervenire. Anzi, giustifica questo atteggiamento deresponsabilizzante dicendosi che ormai il passato è tale e bisogna avere un atteggiamento propositivo per il futuro. Si potrebbe dire che questo direttore si rende complice del misfatto dopo il fatto, ma è più corretto dire che tacendo delle azioni immorali dell'agente, compie un misfatto: è colpevole di omissione, poiché dovrebbe (dal punto di vista morale, se non da quello giuridico) rilevare e denunciare il comportamento del collega, prendere provvedimenti perché ciò non accada più o sanzionarlo (poiché nell'ottica della gestione 'aziendale' della ricerca, il di-rettore gestisce le 'risorse umane') ${ }^{6}$. Il punto è che in certi casi, per esempio quando sono state instaurate relazioni personali di un certo tipo, si rende complice chi facilita una trasgressione impedendo che venga identificata come tale e sanzionata.

\section{Comunione di intenti e legami personali}

Secondo una definizione filosofica particolarmente utile e largamente utilizzata, la complicità comporta una comunione di intenti. Contro la concezione standard causalista, Christopher Kutz sostiene che la relazione di complicità non è necessariamente di tipo causale ${ }^{7}$. Si tratta di un modello utile per mettere a fuoco interazioni nelle quali l'agire collettivo è distribuito e sostiene che atti di aiuto e incoraggiamento costituiscono forme paradigmatiche della complicità. Ciò accade in contesti nei quali non è facile rintracciare una linea causale precisa e i fenomeni di sotto-determinazione o sovra-determinazione causale sono frequenti. D'altra parte, almeno in certi casi, per l'accusa di complicità è sufficiente determinare che vi sia stato un contributo 'potenzialmente causale', ovvero, che il complice abbia contribuito all'agire dell'agente in un modo che avrebbe potuto avere effetti decisivi, anche se non li ha avuti. Un esempio paradigmatico a sostegno di questa tesi è quello del ruolo dei funzionari legali americani nel favoreggiamento di forme illegali di interrogatorio dopo il 2001. Secondo Kutz è l'intenzione a partecipare in un'azione collettiva (ingiusta) che stabilisce la complicità,

L'esempio del complice che si rende complice 'coprendo' l'agente, vd. G. Mellema, Complicity and Moral Accountability, cit., pp. 68, 134.

7 C. Kutz, Complicity: Ethics and Law for a Collective Age, Cambridge University Press, Cambridge 2000. 
anche se dal punto di vista causale la sua azione non dà un contributo causale oppure è sovra-determinata e quindi ridondante ${ }^{8}$. Si può partecipare all'azione criminale, senza dare un contributo di tipo causale, ma intenzionale.

Vista la centralità che questa teoria ha acquisito si può obiettare che la conclusione che ho tratto sopra è affrettata, poiché la definizione di Kutz sembra subordinare la complicità intesa come concorso effettivo e performativo, come contributo causale all'agire di un altro attore, alla complicità intesa come concorso nell'intenzione, per esempio come concorso morale. Ma vorrei presentare alcune ragioni a sostegno del mio scetticismo.

In primo luogo, se si mette l'accento sulle intenzioni, si ritaglia la complicità all'interno dell'agire intenzionale. La condivisione di intenzioni partecipative riconosce una pianificazione complessa e, all'interno di questa, la condivisione esplicita di un obiettivo, che è il fine identificato nella progettazione del piano. La complicità nell'esecuzione del piano deve riconoscere però anche una distribuzione impari delle mansioni, dei ruoli o degli snodi causali che portano al compimento del piano. Infatti, se non ci fosse questa discrepanza, gli agenti non sarebbero semplicemente complici, ma coautori della stessa azione o agenti comprimari, e quindi anche corresponsabili. Il complice deve avere un ruolo secondario nell'esecuzione del piano, anche se questo ruolo può essere decisivo per il successo del piano. Infatti, se il ruolo del complice non fosse determinante, allora la sua implicazione nell'agire dell'agente primario non sarebbe affatto ovvia e l'accusa di responsabilità potrebbe essere del tutto ingiustificata.

Molti casi di complicità dipendono interamente dalla condivisione di un obiettivo e, di conseguenza, di un piano. In questo caso è importante definire la complicità in termini di intenzioni condivise poiché preme far risaltare la condivisione esplicita del piano e la consapevolezza che ciascuno dei complici ha del proprio ruolo e della propria efficacia nell'esecuzione del piano e del ruolo e dell'efficacia dell'altro. Questo è il caso centrale nella teoria dell'azione intenzionale collettiva ${ }^{9}$. La condivisione delle intenzioni è un elemento per molti strutturale dell'azione collettiva in cui ciascun agente ha un ruolo determinante ma non pari. Se il ruolo di ciascuno fosse pari, allora l'azione congiunta sarebbe un esempio di agire intenzionale condiviso. Quindi la complicità, per differenziarsi dall'agire condiviso, deve basarsi

8 C. Kutz, Complicity: Ethics and Law for a Collective Age, cit., pp. 122, 186.

9 M. Gilbert, Complicity: Ethics and Law for a Collective Age, "Philosophy and Phenomenological Research",67, 2003/1, pp. 236-239; L. May, Complicity: Ethics and Law for a Collective Age, "Philosophical Review", 111, 2002/3, pp. 483-486; B. Lawson, Individual Complicity in Collective Wrongdoing, "Ethical Theory and Moral Practice", 16, 2013/2, pp. 227-243. 
sulla disparità di funzioni o di efficacia causale. Entrambi i tipi di azioni si basano sull'impegno reciproco che ciascuno faccia la sua parte ${ }^{10}$.

Ora, ci sono casi di complicità che non prevedono la condivisione di un obiettivo. In certi casi, infatti, gli agenti diventano complici, senza davvero esplicitare o esprimere intenti. Talvolta, si diventa complici per una relazione di fiducia stabilita ben prima dell'atto in questione. Due amici di lunga data possono trovarsi in una relazione di complicità che non è relativa né determinata dalla condivisione di alcun piano. Anzi, gli amici in questione possono di fatto divergere aspramente sulla natura e l'opportunità dell'azione in cui si trovano a essere complici. Il complice può trovarsi ad acconsentire alla partecipazione nell'esecuzione dell'atto per onorare i vincoli di un'amicizia antica, pur disapprovando l'agente primario. In questo caso, il complice non è costretto da una minaccia, né da un compromesso inevitabile, bensì stretto da un legame che è fonte di obblighi e ragioni normative, sebbene non determinanti. Si può dire che il complice agisce suo malgrado, sebbene agisca intenzionalmente e non sia vincolato esteriormente. Diversamente dal caso della manipolazione e del ricatto psicologico, la pressione psicologica e normativa non ha carattere violento ed estorsivo e non è esercitata in modo asimmetrico da uno sull'altro. Piuttosto, è la relazione personale, nella sua storicità, che è sorgente normativa di ragioni.

La relazione personale può costituire un legame fondativo nei gruppi di piccole e medie dimensioni. Essa spiega azioni collettive che sarebbero altrimenti inspiegabili, che sarebbe un errore accantonare come casi di irrazionalità pratica. In certi casi il legame personale costituisce la relazione fiduciaria che è presente nei casi ordinari di azione cooperativa e condivisa, quando questa non è fondata interamente sulla reciproca dipendenza. È un legame che funziona da collante anche in situazioni fortemente asimmetriche, dove può facilmente venire a mancare il rispetto reciproco, ed è compatibile con una quantità di relazioni controverse nelle quali l'agente o il complice intrattengono relazioni problematiche dal punto di vista morale, per esempio nel caso di oppressione, paternalismo o minaccia.

Quando il contributo intenzionale non è presente vi sono due tipi di giustificazioni, una relativa al contributo causale (anche inconsapevole) del complice nella produzione dell'atto, e l'altra relativa alla relazione personale che complice e agente intrattengono. Il vantaggio di questo secondo approccio è che spiega casi in cui il modello causale standard non rende conto del fenomeno cooperativo, nel quale agenti diversi contribuiscono a sovra-

10 Rimangono questioni aperte su come intendere tale impegno reciproco, se sia necessaria una certa forma di fiducia nell'altro a onorare la propria responsabilità rispetto al piano condiviso etc. 
determinare l'azione, oppure i casi in cui il contributo causale specifico è sconosciuto e non conoscibile.

Queste precisazioni impongono cautela nella ricerca di una definizione troppo angusta, che potrebbe escludere dal novero fenomeni importanti, ma scoraggiano anche una definizione ampia o vaga che impedisce una caratterizzazione perspicua di tali fenomeni, nascondendo le peculiarità distintive. In particolare, l'enfasi sulla condivisione delle intenzioni nel perseguimento di un obiettivo comune tra agente e complice, può nascondere diversità importanti che intercorrono tra forme dell'agire cooperativo che dipendono dalla divisione di compiti e complicità ${ }^{11}$. Tornerò su questo punto più avanti.

\section{Complicità di ruolo}

Il modello intenzionalista di Kutz è risultato limitante anche in relazione a certi casi moralmente problematici in cui l'agente è definito da relazioni gerarchiche o di ruolo, difficilmente traducibili nei termini di intenzioni partecipative. In polemica con la teoria di Kutz, Herlinde Pauer-Studer ha sostenuto che la complicità può essere generata dalla dipendenza del ruolo professionale dai principi normativi che costituiscono la struttura di una organizzazione o istituzione delle cui pratiche l'agente si rende complice ${ }^{12}$. In questi casi specifici, l'agente è vincolato da norme che regolano internamente il suo essere agente. Se l'agente si sottraesse a questi vincoli normativi, si dovrebbe parlare di un 'fallimento costitutivo', poiché verrebbe meno a norme che lo costituiscono in quanto agente con una certa identità professionale. L'identità professionale funge da 'identità pratica', nel senso che provvede alla descrizione sotto la quale l'agente si attribuisce valore o si riconosce di valore ${ }^{13}$.

Per illustrare lo statuto profondamente ambiguo della complicità, PauerStuder usa l'esempio di Georg Konrad Morgen, un 'coscienzioso' magistrato e giudice delle SS nazionalsocialiste che 'per amor di giustizia' rifiutò di emettere un verdetto tendenzioso sotto la pressione del partito nazista e fu destituito. Su mandato di Henrich Himmler fu poi incaricato di investigare sulla corruzione interna ai lager e lo fece scrupolosamente, conducendo a

\footnotetext{
11 Su complicità e cooperazione vd. Mellema, op. cit., cap. 9.

12 H. Pauer-Studer, Complicity and Conditions of Agency, "Journal of Applied Philosophy", 35, 2018/4, pp. 643-660.

13 Pauer-Studer interpreta l'esempio di Morgen adottando un approccio costitutivista, secondo il quale la normatività delle ragioni per l'azione è radicata in principi costitutivi del sé.
} 
numerose incriminazioni ${ }^{14}$. In seguito, Morgen venne autorizzato a investigare sulle 'uccisioni arbitrarie' nei campi di sterminio di Treblinka e Majdenek. Durante il processo di Norimberga, sostenne di avere avuto un comportamento moralmente ineccepibile attraverso tutta la sua carriera di giudice.

Ciò che è interessante in questo esempio è che Morgen ritiene di aver adottato e realizzato gli stessi principi di imparzialità prima, durante e dopo il regime nazista, come se il contesto normativo e giuridico nazista fosse una cornice esterna al suo operare. Nel tentativo di giustificare la sua complicità e sottrarsi alla condanna morale, Morgen sostiene anche di aver combattuto il sistema nazista, con mezzi leciti e morali, cercando di combattere la criminalità al suo interno, mostrando con ciò di trattare il sistema normativo nazista come un fatto circostanziale che non inquina l'agire individuale. La sua tesi è che l'attività legale ha principi costitutivi di imparzialità che rimangono intatti pur attraversando circostanze storiche del nazismo e della liberazione.

Secondo Pauer-Studer l'esempio di Morgen è paradigmatico e porta alla luce la complessità dell'ambivalenza morale che risulta dalle condizioni nonideali in cui operano agenti limitati, condizioni nelle quali le distinzioni ordinarie tra giusto e ingiusto sono confuse e, soprattutto, il vocabolario concettuale della moralità è posto al servizio di un sistema normativo profondamente distorto. La giustificazione di Morgen è disturbante non perché Morgen si nasconde dietro i rapporti gerarchici e si appella all'inderogabilità degli ordini, ma proprio perché fa sua l'etica dell'onore e dell'imparzialità e, all'interno di un sistema normativo arbitrario e massimamente ingiusto, si mette alla caccia di uccisioni arbitrarie e ufficiali corrotti. La conclusione di Pauer-Studer è che in situazioni non-ideali i principi morali non funzionano più come argini e barriere contro l'effetto corrosivo del male; e ciò indipendentemente dalla questione se sia possibile e opportuno distinguere tra positività della legge e la morale, un dibattito che ha appassionato i giuristi e filosofi morali del dopoguerra. La realtà in cui opera Morgen è molto più complessa e l'ambiguità delle sue giustificazioni non è solo autoassolutoria. In condizioni così compromesse, le distinzioni morali non sono più affidabili. Morgen difende l'operato delle SS in quanto 'a norma di legge' e garante di uno stato di diritto avvalendosi di concetti morali fraintesi e distorti. L'ambiguità morale è radicale, nel senso che interessa i concetti stessi che articolano la moralità. 


\section{Condizioni non-ideali e circostanze attenuanti}

Nell'autodifesa di Morgen, traspare un argomento che ritroviamo spesso nella letteratura sulla complicità, che si fonda sulla distinzione tra condizioni ideali e non-ideali. Morgen dichiara di aderire ai principi morali fondamentali ma ammette che ci sono limiti importanti a ciò che un individuo può fare in condizioni di ingiustizia pervasiva e legalizzata. Consapevole di questi limiti, Morgen decide di combattere il regime nazista dall'interno, ovvero indagando su crimini e misfatti, come la corruzione del sistema carcerario dei lager e le uccisioni arbitrarie ${ }^{15}$. Morgen si appella ai principi morali e anche alla consapevolezza dei limiti individuali. La sua complicità con il regime nazista è giustificata da un argomento morale e la caratterizzazione del proprio ruolo professionale è fortemente moralizzata, cioè definita nei termini della fedeltà ai principi morali di imparzialità, decenza, e onestà; la virtù è intesa come resistenza alla corruzione e all'appropriazione indebita. Pauer-Studer denuncia il carattere radicalmente ambiguo di questo appello ma non mette in discussione l'argomento del doppio standard invocato da Morgen.

In effetti, è un argomento che ritroviamo nella letteratura kantiana recente, dove il problema della complicità è affrontato principalmente in relazione al dibattito sul rigorismo dell'etica universalista kantiana. Entro questo dibattitto si fa ricorso alla distinzione tra le condizioni ideali e le condizioni non ideali della teoria normativa, ma ci sono modi diversi di intendere questa distinzione. Nella sua formulazione originaria, John Rawls identifica due condizioni: la condizione di conformità (compliance) parziale e le «limitazioni naturali e contingenze storiche ${ }^{16}$. In condizioni di piena conformità alla norma (full compliance) le violazioni ci sono, ma contano come eccezioni. Il problema è se questa discrepanza modifica le responsabilità individuali e altera la normatività dei principi morali; in altre parole, questa distinzione suggerisce un doppio standard?

Secondo Liam Murphy, le responsabilità morali che abbiamo in condizioni non-ideali sono le stesse di quelle che abbiamo in condizioni ideali ${ }^{17}$. Da questo punto di vista, allora, Morgen è complice del sistema nazista e le sue giustificazioni sono inaccettabili poiché è vincolato dagli stessi principi morali che governano una comunità di agenti razionali in condizioni ideali.

\footnotetext{
15 Pauer-Studer riporta brani significativi dell'interrogatorio, vd. Law and Morality under Evil Conditions: The SS Judge Konrad Morgen, "Jurisprudence”, 3, 2012/2, pp. 367-390, p. 383.

16 J. Rawls, A Theory Justice, Harvard University Press, Cambridge 1971, sez. 2, 39, p. 246.

17 L. Murphy, Moral Demands in Nonideal Theory, Oxford University Press, Oxford 2000. Murphy definisce le condizioni non-ideali come quelle in cui il comportamento non è conforme alla norma (noncompliance).
} 
Per Christine Korsgaard, invece, le condizioni non-ideali sono condizioni alle quali il tentativo di rispettare la legge morale nella sua forma più stringente trasformerebbe l'agente ben intenzionato in uno «strumento del male» ${ }^{18}$. Questa definizione è usata per giustificare un'etica più permissiva, ovvero, una interpretazione della legge morale che si adatti alle circostanze non ideali in cui gli agenti umani operano. Si tratterebbe, dunque, di un adattamento ai limiti della razionalità umana, le cui aberrazioni sono visibili sia a livello dell' agire individuale, sia al livello dell'agire collettivo. L'agente morale ha il dovere di «prendere la legge nelle sue mani», ovvero di farsene interprete, nel contesto in cui l'azione diventa urgente ${ }^{19}$. Così, l'agente che mente al nazista a proposito del nascondiglio degli ebrei fuggitivi, non fa violenza alla legge morale, ma adatta la legge morale alle peculiarità delle circostanze storiche. Facendo seguito a questo argomento, Tamar Schapiro ha sostenuto più precisamente che il concetto di condizione ideale equivale al concetto di 'circostanza attenuante' poiché ha l'effetto di mitigare la stringenza di principi morali e politici che si applicano in condizioni ideali ${ }^{20}$.

Questo approccio vanta il merito di rendere intelligibile l'autodifesa di Morgen e quindi spiegare la struttura normativa e motivazionale di un ampio spettro di fenomeni che si possono associare a questo esempio paradigmatico, non solo guardando alla Germania e all'Italia fascista, ma generalizzando alle società cosiddette transizionali. Tuttavia, ritengo che questo merito esplicativo sia solo apparente ${ }^{21}$.

In primo luogo, nello specifico della letteratura kantiana, questa interpretazione rende oscuro il ruolo del requisito di universalità, non solo come garanzia di coerenza e oggettività dei principi morali, ma anche come principio strutturale dell'agire razionale ${ }^{22}$. Infatti, in una prospettiva kantiana,

18 C. Korsgaard, The Right to Lie: Kant on Dealing with Evil, in J. Rachels (ed.), Ethical Theory 2: Theories About How We Should Live, Oxford University Press, Oxford 1998, p. 153.

19 C. Korsgaard, Taking the Law into Our Own Hands: Kant on Right to Revolution, in A. Reath, B. Herman, and Korsgaard, eds., Reclaiming the History of Ethics: Essays for John Rawls, Cambridge University Press, Cambridge 1997, pp. 297-328.

20 T. Schapiro, Compliance, Complicity, and the Nature of Nonideal Conditions, "Journal of Philosophy", 100, 2003/7, pp. 329-355. Schapiro sostanzialmente riprende la teoria elaborata da Korsgaard in Taking the Law into Our Own Hands: Kant on Right to Revolution, sebbene si ponga in modo più critico rispetto ad un saggio precedente di Korsgaard, nel quale l'impatto della distinzione tra condizioni ideali e nonideale viene caratterizzato in modo differente, vd. The Right to Lie: Kant on Dealing with Evil, cit. 21 Secondo Michael Cholbi il resoconto di Schapiro - e quindi anche quello di Korsgaard - non può spiegare le differenze sostanziali tra la permissibilità della menzogna difensiva e altre forme di azione più dubbie dal punto di vista morale, vd. The Constitutive Approach to Kantian Rigorism, "Ethical Theory and Moral Practice”, 16, 2013, pp. 439-448, p. 445. Anche Robert Jubb sottolinea la difficoltà di ricavare principi morali validi in situazioni non ideali, vd. Norms, evaluations, and ideal and nonideal theory, "Social Philosophy and Policy", 33, 2016, pp. 393-412, p. 400.

${ }^{22}$ Per l'argomento a sostegno di questa interpretazione rimando a C. Bagnoli, The practical significance of the categorical imperative, "Oxford Studies in Normative Ethics", 11, 2021, in via di pubblicazione. 
l'universalità dei principi morali rende conto di un certo tipo di integrità etica e metafisica e anche di stabilità diacronica. Sostenere che i principi morali hanno una stringenza normativa differente quando applicati in condizioni non-ideali solleva questioni importanti sull'integrità e sulla stabilità diacronica di agenti razionali che operano in condizioni non-ideali. L'implicazione di questo approccio sembra essere che il requisito di universalità non è sufficiente a garantire l'integrità, l'autogoverno normativo e la stabilità diacronica in condizioni non-ideali. Se in tali condizioni non-ideali l'integrità e la stabilità non sono garantite dall'universalità dei principi, da che cosa sono garantite?

Questa domanda apre un dibattito su vari fronti: sulla natura e il ruolo del requisito di universalità, e sulla forza dell'argomento costitutivista che è presupposto dalle trattazioni di Korsgaard e Pauer-Studer, secondo il quale la normatività delle ragioni per l'azione dipende da norme costitutive dell'azione razionale e costitutive del sé dell'agente razionale, nello specifico, il requisito di universalità. Modulare il requisito di universalità secondo le condizioni non-ideali significa anche riconoscere che la normatività delle ragioni per l'azione concreta non è spiegata interamente dal costitutivismo. È un dibattito che ci porta altrove ${ }^{23}$. Qui mi preme mettere in luce perché l'argomento di Schapiro non aiuta a comprendere meglio il fenomeno della complicità.

\section{Complicità e implicazione: le responsabilità dell'interdipen- denza in condizioni di ingiustizia sistemica}

Il dibattito filosofico si è concentrato sulla complicità come una forma di concorso intenzionale in attività criminale o immorale, come risulta evidente dalla letteratura sulle condizioni non-ideali. Eppure l'esempio di Morgen illustra in modo drammatico alcuni elementi della relazione di complicità che non sono limitati al concorso diretto e intenzionale in attività immorali, ma sono inevitabili in condizioni di ingiustizia sistemica.

Il primo elemento riguarda il carattere gerarchico dell'ordinamento in cui Morgen si riconosce. Infatti, Morgen deve ammettere che il fallimento dei

\footnotetext{
23 Sulla centralità dell'argomento costitutivista nella discussione della normatività in una prospettiva kantiana, vd. C. M. Korsgaard, Constitutivism and the virtues, "Philosophical Explorations", 22, 2019/2, pp. 98-116, C. Bagnoli, Authority as a Contingency Plan, "Philosophical Explorations", 2019 22/2, pp. 130-145. Sui limiti generali dell'argomento costitutivista in sostegno della normatività robusta, vd. L. Ferrero, The Simple Constitutivist Move "Philosophical Explorations", 22, 2019/2,pp. 146-162; sui limiti dell'argomento costitutivista kantiano, vd. W. Gobsch, Autonomy and radical evil: a constitutivism, "Philosophical Explorations", 22, 2019/2, pp. 194-207. Per una critica della concezione di Korsgaard del requisito di universalità (stretta e provvisoria), vd. C. Bagnoli, Defeaters and Practical Knowledge, "Synthese", 195, 2018/7, pp. 2855-2875.
} 
suoi 'tentativi di resistenza' è dovuto principalmente al modo in cui viene trasmessa l'autorità nei regimi totalitari, attraverso una stretta relazione gerarchica che non prevede bilanciamento. Ora, la struttura gerarchica non è una invenzione del regime nazista ed è presente nelle forme complesse della divisione del lavoro. In certe attività, la distribuzione del lavoro è più efficace se è ordinata in modo gerarchico (p.e. attraverso l'identificazione e la distribuzione di competenze e poteri), anziché p.e. negoziata, oppure costruita attraverso procedure deliberative. La negoziazione e la costruzione deliberativa sono modalità di distribuzione dei compiti ben più faticose, lente e dispendiose. Esse hanno il vantaggio di preservare un certo tipo di relazione personale tra gli agenti che contribuiscono all'azione. Queste sono le modalità distintive delle istituzioni democratiche proprio perché presumono che l'agente individuale partecipi in modo responsabile dell'attività cui contribuisce. Ma non tutti i casi di distribuzione del lavoro possono avvenire in modo procedurale e deliberato. In alcuni casi, la distribuzione del lavoro si combina con sistemi di delega che compartimentalizzano le responsabilità individuali. Pensiamo, per esempio, all'azione di un operaio alla sofisticata catena di montaggio di una industria altamente tecnologica di cui non conosce nei particolari il processo produttivo, né l'oggetto finale, né il committente e soprattutto non sa a quale punto del processo interviene. La sua responsabilità individuale pare limitata a un segmento molto particolare del processo produttivo. Questo agente non è completamente consapevole dell'azione che compie ed è all'oscuro delle responsabilità più ampie e gravose che gli sono imputabili sulla base del fatto che, volente o nolente, è implicato in un processo produttivo ben più vasto.

Supponiamo che l'industria per cui lavora contribuisca a produrre sostanze di scarto tossiche. Questa implicazione è sufficiente a renderlo responsabile per l'impatto ambientale della produzione industriale? La mancanza di consapevolezza di ciò che fa gli può essere contestata. In contesti fortemente gerarchici, l'agente individuale è (almeno parzialmente) alienato dalla propria azione ed è proprio questa alienazione parziale che consente il 'buon funzionamento' del sistema ${ }^{24}$. Ma ci sono responsabilità (morali) che

\footnotetext{
24 Sull'alienazione nei sistemi di azione collettiva, vd. S. Shapiro, Massively Shared Agency, in Rational and social agency: The philosophy of Michael Bratman, Oxford University Press, 2014, pp. 257-293; D. Plunkett, \& S. Shapiro, Law, Morality, and Everything Else: General Jurisprudence as a Branch of Metanormative Inquiry, "Ethics", 128, 2017/1, pp. 37-68; S. Shapiro, Authority, in J. Coleman \& S. J. Shapiro, eds., The Oxford Handbook of Jurisprudence \& Philosophy of Law, Oxford University Press, 2002. Cfr. C. Bagnoli, One Among Many: Responsibility and Alienation in Mass Action, in T. Marquez \& C. Valentini (eds.), Collective Action, Philosophy and the Law, Routledge, London, in via di pubblicazione.
} 
non sono delegabili. Queste responsabilità sussistono, anche se l'agente non se ne rende conto.

Il secondo elemento generalizzabile dell'esempio di Morgen è proprio il carattere della sua complicità che egli ritiene circostanziale, ovvero, il fatto che ciò che un agente individuale può fare o meno, le opportunità di agire $\mathrm{e}$ i modi in cui si trova a esercitare la sua razionalità pratica non dipendono interamente dalla sua intenzione di partecipare attivamente a una pratica o di contribuire attivamente all'azione di una certa istituzione. L'agire degli esseri umani è fortemente interattivo e interdipendente. Anziché circostanziale, questo è un elemento strutturale dell'agire umano, nonché una delle forme costitutive della razionalità pratica. Ma proprio per questa caratteristica strutturale e costitutiva del suo agire, l'agente umano è sempre implicato nell'azione collettiva ${ }^{25}$.

Questa implicazione è una forma di compromissione morale? A quali condizioni può essere imputata all'individuo come una colpa ${ }^{26}$ ? Come si è visto, vi sono risposte diverse a questa domanda. I consequenzialisti guardano al contributo causale. Per contro, Kutz costruisce una concezione della complicità a partire dall'analisi dell'azione collettiva e dell'intenzione individuale. La tesi principale è che l'agente complice è responsabile perché e nella misura in cui partecipa intenzionalmente all'azione collettiva, indipendentemente dal fatto che questa sua partecipazione abbia un effetto, ovvero, faccia una differenza dal punto di vista causale. La ridondanza causale non è una scusante dal punto di vista morale.

Ma se è vero che siamo continuamente chiamati in causa dall'azione degli altri, impigliati nell'operare perverso o distorto di certe realtà istituzionale, l'agire individuale è moralmente compromesso in modo radicale, ben aldilà delle azioni motivate da intenzioni partecipative. In condizioni di ingiustizia sistemica nelle quali operiamo, l'agente individuale è costretto ordinariamente a scelte morali dilemmatiche e non può mai dirsi innocente ${ }^{27}$. Basta pensare ai macro-fenomeni come il riscaldamento globale e la strage

\footnotetext{
25 R. Jay Wallace ritiene che ci siano situazioni in cui agenti ordinari e motivati moralmente sono implicati in relazioni sistemiche dove le disparità sociali ed economiche sono marcate, loro malgrado, vd. The View from Here: On Affirmation, Attachment and the Limits of Regret, Oxford University Press, Oxford 2013,p. 187. Questa implicazione si estende a tutte le relazioni storiche contingenti, in situazioni di ingiustizia strutturale e istituzionalizzata.

26 Secondo Saba, l'agente è imputabile per misfatti compiuti dal complice nella misura in cui lo autorizza ad agire secondo le sue richieste, vd. Saba Bazargan-Forward, Complicity, in M. Jankovic \& K. Ludwig eds., Routledge Handbook on Collective Intentionality, Routledge University Press, 2017.

27 In dialogo con Shapiro e Korsgaard, Williams sostiene con forza l'ipotesi della complicità inevitabile in condizioni di ingiustizia sistemica, nelle quali l'agente individuale non può essere innocente, $\mathrm{G}$. Williams, The Social Creation of Morality and Complicity in Collective Harms: A Kantian Account, "Journal of Applied Philosophy", 36, 2019/3, pp. 457-470.
} 
degli immigrati clandestini, per accorgersi con orrore che si tratta di complicità inevitabili. Queste azioni collettive di vasta scala hanno tutte la stessa struttura, che sfrutta l'interdipendenza costitutiva degli agenti umani. La complicità inevitabile è un fatto che ci espone in tutta la nostra limitatezza, incapaci di prenderci le responsabilità che ci spettano. Essa pone in modo urgente la necessità di riformare sostanzialmente il modo in cui abitiamo il mondo. La diagnosi di ingiustizia sistemica non discolpa, ma approfondisce e allarga l'accusa di complicità.

Questa analisi fa emergere che la complicità inevitabile è l'altra faccia dell'interdipendenza. Così come siamo implicati inevitabilmente nell'agire dell'altro, possiamo anche implicare gli altri in azioni congiunte di riparazione e riforma ${ }^{28}$. Prendere sul serio la necessità della riparazione e della riforma impegna a richiamarsi in modo rigoroso all'universalità dei principi morali basilari volti a proteggere la collettività attraverso il sistema di responsabilità reciproche. Non è attenuando il rigore dei principi morali che possiamo far fronte alle responsabilità che ci investono, ma cercando di mettere a frutto le capacità di razionalità pratica di cui siamo dotati. Qui possiamo utilmente tornare agli esempi di complicità all'inizio di questa discussione, proprio per illustrare alcune di queste capacità all'opera. L'urgenza della riforma istituzionale e del riorientamento morale può essere affrontata ripensando i principi morali universali come principi di razionalità pratica, fruibili da agenti limitati e interdipendenti ma anche dotati di una sensibilità capace di organizzare salienze condivise.

\footnotetext{
28 La riparazione morale è un concetto fondamentale per la comprensione del sistema della responsabilità, vd. E. Peck \& E. K. Feder, Institutional Evils, Culpable Complicity, and Duties to Engage in Moral Repair, "Metaphilosophy", 48, 2017/3, pp. 203-226; C. Bagnoli, Teoria della Responsabilità, Il Mulino, Bologna 2019, cap. 12.
} 